\title{
E stado actual de la resistencia de Helicobacter pylori a tetraciclina: revisión sistemática de la literatura
}

\author{
Paola Ramírez-Bulla ${ }^{1}$, M arcela M ercado-R eyes ${ }^{1}$, Alba Alicia Trespalacios-R angel ${ }^{1}$, \\ J enny A vila-C oy'; W illiam Otero-R egino² \\ ${ }^{1}$ Grupo de Enfermedades Infecciosas. Departamento de M icrobiología. Facultad de Ciencias. Pontificia U niversidad J averiana. \\ Bogotá, D.C., Colombia. \\ 2 U nidad de Gastroenterología, U niversidad N acional de Colombia, B ogotá, D.C., Colombia \\ * alba.trespalacio@javeriana.edu.co
}

Recibido: 23-05-2012; A ceptado: 13-07-2012

\section{Resumen}

Objetivo. Determinar la prevalencia de resistencia de Helicobacter pylori a tetraciclina y las posibles mutaciones que generan esta resistencia mundialmente. Materiales y método. Se realizó una búsqueda sistemática de literatura en las bases de datos: M edline, Science Direct (Elsevier), Ovid, Pubmed, Lilacs y MEDICLATINA, con el uso de palabras clave relevantes. La extracción de los datos fue independiente y se realizaron listas de verificación para evaluar la calidad metodológica de los estudios. El análisis de la información fue realizado en el programa RevM an $5 \circledR$. Resultados. Se evidenció resistencia a tetraciclina por Helicobacter pylori con prevalencias de 1\% para N orte A mérica, $8 \%$ para Centro y Sur A mérica; $5 \%$ para A sia y 2\% para Europa. La alta resistencia se debe a la triple mutación A GA (926-928)-->TTC, en el gen 16S rD N A. Conclusiones. La resistencia antibiótica es una de las causas que más se asocia a falla terapéutica en la erradicación de Helicobacter pylori, así como la poca adherencia al tratamiento y el uso indiscriminado de antibióticos. Se evidenció que la tasa de resistencia a tetraciclina aumenta después de un primer tratamiento con este antibiótico, sin embargo la prevalencia de la resistencia global a tetraciclina es baja sin aún alcanzar límites que impidan su utilización en los esquemas de tratamiento.

Palabras clave: H elicobacter pylori, tetraciclina, resistencia, mutación, tratamiento, epidemiología, 16S rR NA.

\begin{abstract}
C urrent status of Helicobacter pylori resistance to tetracycline: a systematic review of the literature. 0 bjective. To determine the prevalence of Helicobacter pylori resistance to and the possible mutations that generate this worldwide resistance. Materials and methods. A systematic search for literature was performed in the databases M edline, Science Direct (Elsevier), O vid, PubM ed, Lilacs and M edicL atina using relevant key words. D ata extraction was independent and checklists were prepared to assess the methodological quality of the studies. A nalysis of information was done with R evM an $5 \AA$. Results. We found H elicobacter pylori resistance preval ence rates of $1 \%$ for N orth A merica, $8 \%$ for Central and South A merica, $5 \%$ for A sia, and $2 \%$ for Europe. The mutation associated to this resistance is in the 16S rD N A gene at nucleotide position 967TTC to A GA 965 responsible of high resistance to tetracycline. Conclusions. A ntibiotic resistance is one of the causes most associated to treatment failure in the eradication of H elicobacter pylori, as well as poor adherence to treatment and indiscriminate use of antibiotics. W e also evidenced that the rate of tetracycline resistance is higher when it is used in a second treatment scheme. The distribution of resistance is variable in different areas and it is important to know these resistances to avoid treatment failures.
\end{abstract}

Key words: H elicobacter pylori, tetracycline, resistance, mutation, treatment, epidemiology, 16S rR NA. 


\section{Resumo}

E stado atual da resistência do Helicobacter pylori à tetraciclina: uma revisão sistemática da literatura. 0 bjetivo. Determinar a prevalência da resistência do Helicobacter pylori à tetraciclina e possíveis mutações que geram esta resistência a nível mundial. M ateriais e métodos. Foi realizada uma procura sistemática da literatura nas bases de dados M edline, Science Direct (Elsevier), O vid, Pubmed, Lilacs e M EDICLA TIN A, usando palavras-chave relevantes. A extração dos dados foi independente e real izaram-se listas de verificação para avaliar a qualidade metodológica dos estudos. A análise dos dados foi realizada em REV M AN $5 \circledR$. R esultados. Foi evidenciada a resistência de $\mathrm{H}$ elicobacter pylori com taxas de prevalência de 1\% para a A mérica do $\mathrm{N}$ orte, 8\% para A mérica Central e A mérica do Sul; 5\% para Á sia e 2\% para Europa. A mutação associada a estas resistências no gen 165 rDN A nos nucleótidos da posição A GA 965 a 967TTC é responsável da alta resistência à tetraciclina. Conclusões. A resistência aos antibióticos é uma das razões mais associadas à fal ha do tratamento na erradicação de Helicobacter pyl ori, assim como a pouca adesão ao tratamento e uso indiscriminado de antibióticos; também foi evidente que a taxa de resistência à tetraciclina é maior quando utilizado em regime de segundo tratamento; a distribuição da resistência varia em diferentes áreas e é importante saber estas resistências a fim de evitar fal has terapêuticas.

Palavras-chave: Helicobacter pylori, tetraciclina, resistência, mutação, tratamento, epidemiologia, 16S rRNA.

\section{Introducción}

La bacteria Helicobacter pylori es un organismo microaerofílico, Gram negativo, ureasa, catalasa y oxidasa positivos, de crecimiento lento, en forma de espiral, con dos a seis flagelos que le dan la motilidad necesaria para soportar el peristaltismo gástrico y penetrar en la mucosa del estómago $(1,2)$. Se estima que infecciones por Helicobacter pylori ocurren en aproximadamente el $50 \%$ dela población mundial y está asociada al desarrollo de diferentes enfermedades gastroduodenales (3). La infección se puede adquirir durante la infancia y el microorganismo puede persistir en la mucosa gástrica durante toda la vida si no es tratado. $\mathrm{H}$. pylori causa gastritis crónica posterior a la infección inicial, puede permanecer silente durante mucho tiempo debido al equilibrio dinámico entre la bacteria y su hospedero, 0 desarrollar enfermedades más graves (1). A proximadamente el $10 \%$ de los individuos infectados, desarrolla ulcera péptica; del 1 al 3\% desarrolla adenocarcinoma gástrico y menos del $0,1 \%$ linfoma gástrico tipo M A L T (4).

La A gencia Internacional para la Investigación del Cáncer (IARC), demostró la asociación entre la infección por $H$. pylori y cáncer gástrico. A pesar de que se ha observado una disminución general de la incidencia de este cáncer, sigue siendo el cuarto tipo más común y la segunda causa de muerte por cáncer en todo el mundo (5). Datos del M inisterio de la protección Social y del Instituto de Cancerología de Colombia indicaron para el año 2009, 342 casos nuevos de cáncer de estómago, representando el 6,3\% de todos los tipos de cáncer reportados para este año, antecedido por cáncer de cuello uterino, cáncer de próstata y cáncer de glándula tiroides (6).

Independientemente de la histología, la mayoría de cánceres gástricos, presentan infección por $\mathrm{H}$. pylori, estos tumores muy rara vez surgen de la mucosa gástrica sin inflamación. Por lo tanto, H. pylori se ha considerado como factor altamente relacionado con el desarrollo de cáncer gástrico (7). De igual manera, la mayoría de cepas de H. pylori expresan factores de virulencia que se han desarrollado para afectar las vías de señalización celular del hospedero. Entre las muchas características únicas de $\mathrm{H}$. pylori, una de las más notables es su capacidad de sobrevivir durante décadas en el ambiente gástrico, debido a la incapacidad del hospedero para eliminar la infección y al metabolismo de la urea, que por medio de la ureasa produce dióxido de carbono y amonio generando un ambiente neutro que envuelve a la bacteria (4).

L a erradicación de H . pylori disminuye el riesgo de cáncer gástrico en individuos infectados sin lesiones pre-malignas, esto ha sido demostrado con estudios en cohortes de seguimiento en los que la erradicación de H. pylori reduce significativamente el desarrollo de lesiones malignas (8).

Las principales razones del fracaso en la erradicación de la infección por $H$. pylori incluye la resistencia a los antibióticos y la pobre adherencia al tratamiento (9). Las tasas de resistencia varían notablemente en diferentes áreas geográficas por lo tanto la selección de regímenes terapéuticos necesita ajustes de acuerdo a los patrones de resistencia local. L a prevalencia de resistencia a anti bióticos en varias regiones se asocia con el uso general de antibióticos en la región (10). Entre los tratamientos de erradicación de $\mathrm{H}$. pylori, se encuentra el esquema de primera línea que está compuesto por un inhibidor de la bomba de protones, claritromicina y amoxicilina o metronidazol, este esquema es más efectivo si se extiende por más de siete días. Debido al aumento de la resistencia a los antibióticos usados en el tratamiento de primera línea, es necesario replantear el uso de este esquema en diferentes regiones (11).

El esquema de rescate en caso de fracaso del primer tratamiento es el esquema cuádruple que consiste en un inhibidor de la bomba de protones, más citrato de bismuto, 
tetraciclina y metronidazol con eficacia media del $80 \%$. De haber un segundo fracaso, es necesario realizar cultivo de $\mathrm{H}$. pylori para conocer su sensibilidad antimicrobiana. Dentro de los antibióticos en el esquema de tercera línea están levofloxacina, furazolidona, rifabutina y azitromicina, siendo levofloxacina el mejor documentado y se recomienda su uso con amoxicilina más el inhibidor de la bomba de protones $(12,11)$.

En el esquema cuádruple de erradicación de H. pylori, tetraciclina es un anti biótico fundamental (13). Sus val ores de Concentración M ínima Inhibitoria (CM I) general mente reportados están entre 0,25 y $2 \mathrm{mg} / \mathrm{L}$ y la resistencia al antibiótico está por encima de 4 mg/L de CMI (14). Uno de los antibióticos que muestra menor tasa de resistencia primaria es tetraciclina (15). Este es un inhibidor de la síntesis de proteínas que tiene actividad contra las bacterias Gram positivas y Gram negativas (16), se une a la subunidad 30S-ribosomal y bloquea la unión de aminoacil-tRNA, evitando la formación de las cadenas peptídicas (3). Se han propuesto varios factores de resistencia de $\mathrm{H}$. pylori a tetracicl ina como son absorción del antibiótico, inactivación enzimática de la tetraciclina e incremento de bombas de expulsión, sin embargo, el mecanismo más estudiado es la mutación en el gen 165 rDNA (17).

El gen 165 rD NA, es un componente de la subunidad 305 ribosomal, el cual es el blanco de la tetraciclina. Diferentes investigaciones han identificado una triple mutación para adqui rir esta resistencia y una sustitución de los nucleótidos A GA (926-928)-->TTC responsable de los al tos niveles de resistencia de $\mathrm{H}$. pylori a tetraciclina (18). La resistencia bacteriana a tetraciclina, aunque no es tan alta, parece ir en aumento. L as cepas resistentes muestran un rango amplio de CM I (2-256 mg/L) (22). Estudios recientes han identificado de 2 a 6 sitios probables en la interacción ribosomal de al ta afini dad a los antibióticos, mientras que otras investigaciones bioquímicas reportan sitios múltiples de unión de baja afinidad con CMI menores o iguales a 4 mg/L (19).

A demás de las fallas terapéuticas por mutaciones genéticas que presenta la bacteria para hacer resistencia a tetraciclina, existen otras que son explicadas por falta de adherencia al medicamento además de condiciones del huésped, relacionadas con la absorción del antibiótico. L os datos de resistencia reportados son bajos para Europa (2,1\%), A sia $(2,4 \%)$ y A mérica $(2,7 \%)$. Sin embargo las tasas más altas se han encontrado principalmente en Corea $(8,8 \%)$, Chile $(26,8 \%)$ y Camerún $(49,3 \%),(20)$.

Entre las pruebas de evaluación de susceptibilidad microbiana se incluyen métodos como el E-test y el método de dilución en agar siendo este último, el método de referencia avalado por "Clinical Laboratory Standards I nstitute (CLSI)". Esta prueba está basada en cultivos de $\mathrm{H}$. pylori en los que se determina la susceptibilidad a los agentes antimicrobianos comúnmente usados en la terapia de erradicación, aunque es de elección, no se realiza rutinariamente en el diagnóstico de resistencia del $\mathrm{H}$. pylori por su compleja metodología (21). El método de E-test es al tamente concordante con el método de referencia y está recomendado por la "British Society for Antimicrobial C hemotherapy (BSA C)" (21, 22).

Debido a las múltiples diferencias encontradas sobre la resistencia a tetraciclina en el tratamiento para la erradicación de H. pylori, el objetivo de este trabajo es determinar la prevalencia global de la resistencia de $\mathrm{H}$. pylori reportada en la literatura científica en diferentes áreas geográficas, así como la búsqueda de las posibles causas de esta resistencia.

\section{M ateriales y métodos}

Para la búsqueda de los artículos se real izó una exploración en bases de datos M edline, Science Direct (Elsevier), O vid, Pubmed, Lilacs y M EDICLATINA, fechados desde 1998 a 2011, escritos en español o en inglés. Para la búsqueda se incluyeron palabras clave como: $\mathrm{H}$. pylori, tetraciclina (tetracycline), resistencia (resistance), tratamiento (treatment), epidemiología (epidemiology). No hubo restricción en cuanto a la edad, tipo de resistencia (primaria o secundaria) y las patologías de base de los participantes. Se incluyeron artícul os que: 1) presentaran la prevalencia de la resistencia de $\mathrm{H}$. pylori a tetraciclina y/o que reportaran mutaciones relacionadas con la resistencia. 2) en los cuales la resistencia se hubiera evaluado por métodos de dilución en agar, E-test o técnicas de biología molecular. 3. Estudios con diseño de investigación descriptiva. Se excluyeron 1) reportes de tesis doctorales, literatura gris, revisiones 0 cualquier otro tipo de estudio que no fuera descriptivo, 2) A rtículos en los cuales la resistencia fue evaluada por el método de difusión en disco, 3) estudios en donde no se explica claramente la metodología de las pruebas utilizadas y 4) A rtículos que no describen claramente la resistencia a este antibiótico.

\section{Extracción de datos}

Posterior a la selección de los artículos, los datos se extrajeron en un formato estandarizado. De cada uno de los artículos seleccionados se obtuvieron datos de: sensibilidad antibiótica, tipo de mutaciones encontradas, tipo de pruebas microbiológicas realizadas para evaluar la sensibilidad de las muestras y número de participantes del estudio. Para evaluar la calidad de los artículos se realizó una lista de chequeo basada en los parámetros de la guía para usuarios 
de la literatura médica (23). Dos observadores realizaron de forma independiente y ciega la eval uación de calidad de los estudios, asignándole un puntaje final a cada uno. L os ítems de evaluación de la calidad se muestran en la tabla 1. Una vez seleccionados los artículos que cumplieron los requisitos metodológicos de calidad, de cada artículo se obtuvieron datos de porcentaje de resistencia, en caso que no fueran reportados estos datos por los autores se extrajo la información para hallarlos. Los resultados de cada estudio fueron ingresados al programa RevM an5 ${ }^{\circledR}$ de la Biblioteca Cochrane. La evaluación de la calidad metodológica de los artículos se realizó mediante la comprobación de cumplimiento de parámetros establecidos para una revisión de estudios descriptivos mediante una lista de chequeo. L os desacuerdos fueron resuel tos con la ayuda de dos expertos por medio de discusión de acuerdo a los criterios establecidos.

\section{Resultados}

\section{Extracción de datos}

La búsqueda de la literatura arrojo un total de 60 artículos los cuales fueron sometidos a evaluación por parte de dos evaluadores independientes. De estos, 53 cumplieron los criterios de inclusión previamente establecidos. Se excluyeron los artícul os en los cuales la metodología utilizada no era adecuada y no estaba descrita y en los que la resistencia no se describía claramente. Posteriormente, el número de artícul os incluidos para el análisis en el programa RevM an 5 basado en su título, resumen, metodología y resultados fueron 53, teniendo en cuenta los criterios de inclusión y exclusión señal ados anteriormente. Sin embargo, de los 53 artículos, sólo 39 cumplían con los estándares de calidad (Figura 1).

\section{E valuación de la calidad metodológica}

Como se muestra en la figura 2, la calidad metodológica de los estudios eval uados estuvo en los rangos de "cal idad adecuada" en la mayoría de los aspectos evaluados; en todos los artícul os se describía claramente la metodología de las pruebas utilizadas y el uso de controles estándares para evaluar la validez de las pruebas, 39 artículos reportaron la resistencia o los valores para calcular dicha resistencia, 5 artículos reportaron la mutación evaluada, 12 artículos reportaron una resistencia primaria y 5 reportaron resistencia secundaria, 15 artícul os no describieron que tipo de resistencia evaluada y todos los estudios reportaron las conclusiones hechas por los autores.

\section{Discusión}

La revisión de la literatura demostró que hay mayor prevalencia de resistencia a tetraciclina en Centro y Sur A mérica con $8 \%$ de resistencia, seguido por A sia con $5 \%$, Europa 2\% y Norte A mérica con el $1 \%$.

L as mutaciones asociadas a la resistencia de $\mathrm{H}$. pylori se muestran en la tabla 3. En todos los artícul os se secuenció un fragmento del gen $16 \mathrm{~S}$ rDNA, donde se evidenció cambios en las secuencias nucleotídicas. M utaciones puntuales en las cepas susceptibles a este anti biótico se asocian con bajos niveles de M IC (1 ó 2 mg/L vs., $\geq 4 \mathrm{mg} / \mathrm{L}$ ) (24). A demás,

\section{Tabla I . Ítems para evaluación de la calidad metodológica de los artículos.}

\begin{tabular}{cl}
\hline Ítem 1. & $\begin{array}{l}\text { ¿Se realizó una descripción detall lada de los métodos para realizar las pruebas de susceptibilidad a } \\
\text { tetraciclina? }\end{array}$ \\
\hline Ítem 2. & ¿Se estableció la resistencia de H. pylori a tetraciclina o valores para calcular dicha resistencia? \\
\hline Ítem 3. & $\begin{array}{l}\text { ¿Se estableció la posible mutación asociada con la resistencia y fue reportada mediante una técnica } \\
\text { adecuada? }\end{array}$ \\
\hline Ítem 4. & ¿Fueron precisados los sesgos en el estudio? \\
\hline Ítem 5. & ¿Se utilizaron pruebas controles para evaluar el método utilizado? \\
\hline Ítem 6. & ¿La resistencia reportada a tetraciclina fue primaria? \\
\hline Ítem 7. & ¿La resistencia reportada a tetraciclina fue secundaria o posterior a un tratamiento previo? \\
\hline Ítem 8. & ¿Fueron reportadas las conclusiones hechas por el autor con los datos y/o análisis reportados en la \\
& revisión? \\
\hline
\end{tabular}




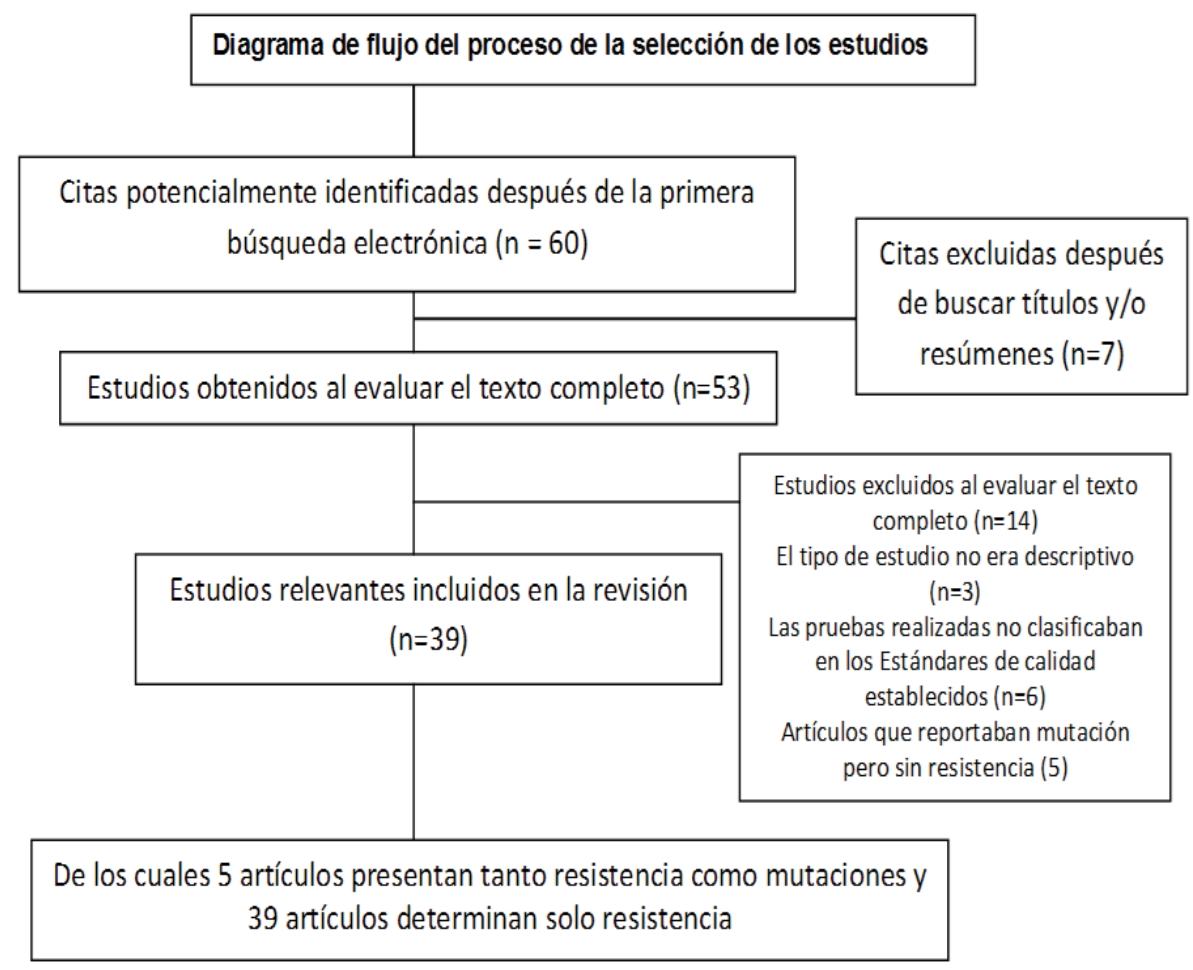

Figura 1: Proceso de selección de los estudios para la revisión sistemática de la literatura.

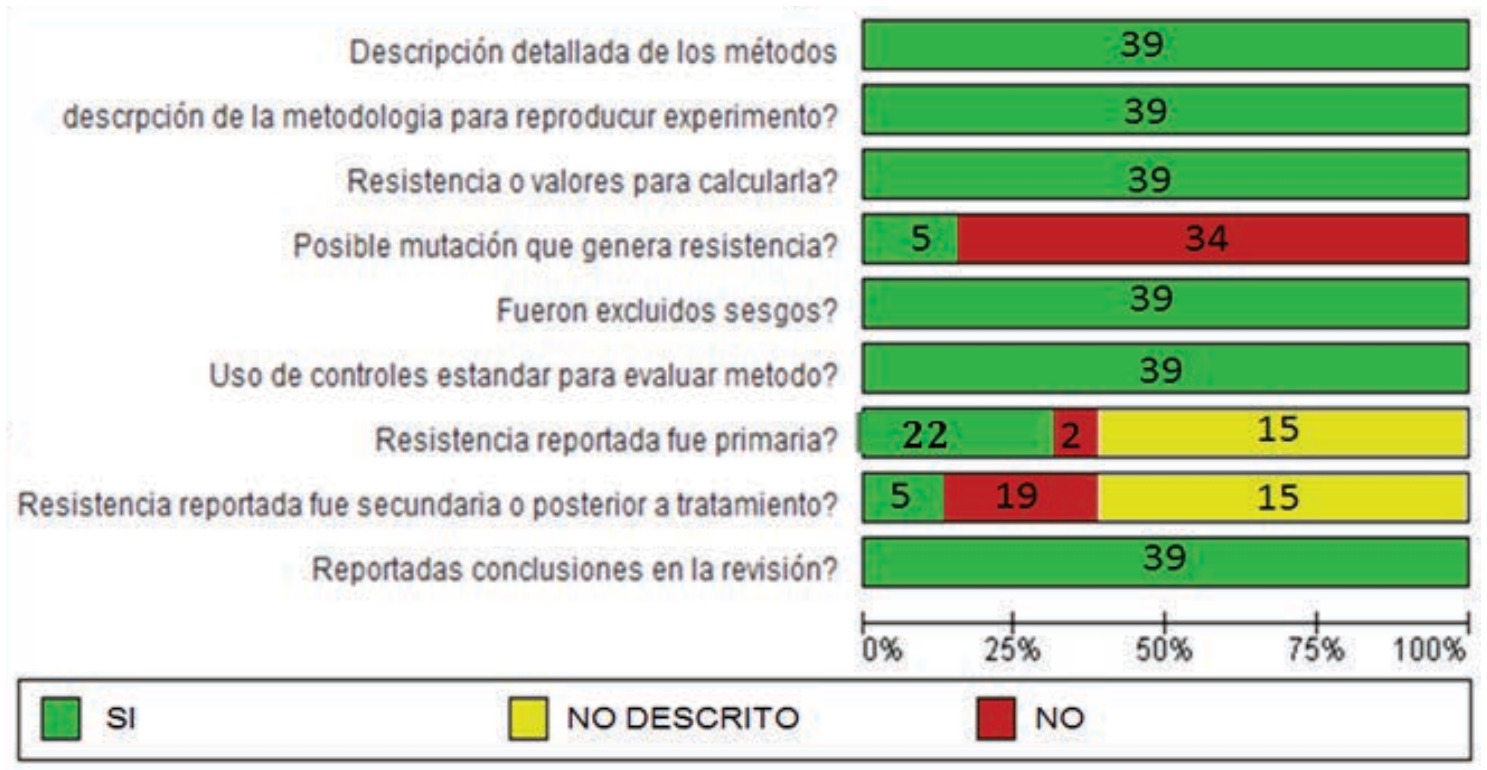

Figura 2. Evaluación de la calidad de los estudios incluidos en la revisión sistemática de la literatura. 
Tabla 2: Frecuencia de resistencia de Helicobacter pylori frente a tetraciclina reportada en diferentes continentes

\begin{tabular}{cccccccc}
\hline Año & $\begin{array}{c}\text { País de } \\
\text { origen }\end{array}$ & Método usado & $\begin{array}{c}\text { No. de } \\
\text { muestras }\end{array}$ & $\begin{array}{c}\text { Tipos de } \\
\text { resistencia }\end{array}$ & $\begin{array}{c}\text { Muestras } \\
\text { resistentes }\end{array}$ & $\begin{array}{c}\% \text { de resistencias } \\
\text { IC: } \mathbf{9 5 \%}\end{array}$ & R ef. \\
\hline 2005 & USA & ADM & 227 & $\begin{array}{c}\text { Primaria y } \\
\text { secundaria }\end{array}$ & 8 & $4 \%$ & $(24)$ \\
\hline 2006 & USA & ADM & 352 & Primaria & 0 & 0 & $(25)$ \\
\hline 2011 & USA & ADM & 531 & No reportada & 0 & 0 & $(26)$ \\
\hline
\end{tabular}

Tasa de resistencia total de Norte América $1110 \quad 8 \quad 0,72 \%$ IC : $(0,2 \%-1 \%)$

\begin{tabular}{|c|c|c|c|c|c|c|c|}
\hline \multicolumn{8}{|c|}{ Sur A mérica y Centro América } \\
\hline 2010 & Chile & ADM & 41 & Primaria & 11 & $26,80 \%$ & (27) \\
\hline 2008 & Cuba & PCR & 28 & No reportada & 3 & 10,7 & (1) \\
\hline 2007 & Chile & ADM & 41 & Primaria & 11 & $26,80 \%$ & (28) \\
\hline 2010 & Cuba & E-test & 40 & No reportada & 0 & 0 & (29) \\
\hline 2009 & Colombia & E-test & 88 & No reportada & 0 & 0 & (30) \\
\hline 2010 & Brasil & E-test & 45 & No reportada & 0 & 0 & (31) \\
\hline 2003 & Brasil & ADM & 155 & No reportada & 13 & $9 \%$ & (32) \\
\hline 2003 & Ecuador & E-test & 42 & No reportada & 0 & 0 & (33) \\
\hline $\begin{array}{l}\text { Tasa de } \\
\text { América }\end{array}$ & stencia total & Sur y Centro & 480 & & 38 & $7,9 \%$ IC : $(5 \%-10 \%)$ & \\
\hline \multicolumn{8}{|c|}{ Asia } \\
\hline 2001 & Corea & ADM & 456 & No reportada & 24 & $5,30 \%$ & (13) \\
\hline 2002 & Libano & E-test & 54 & Secundaria & 1 & $2 \%$ & (34) \\
\hline 2006 & Japon & ADM & 25 & Secundaria & 0 & 0 & (35) \\
\hline 2007 & Iran & E-test & 69 & No reportada & 2 & $2,90 \%$ & (36) \\
\hline 2010 & China & ADM & 293 & Primaria & 1 & $2,93 \%$ & (37) \\
\hline 2010 & Corea & $A D M-P C R$ & 222 & Primaria & 57 & $25,70 \%$ & (38) \\
\hline 2002 & Israel & E-test & 138 & No Reportada & 0 & 0 & (39) \\
\hline 2011 & China & E-test & 73 & Primaria & 0 & 0 & (40) \\
\hline 2011 & Irán & ADM & 42 & Secundaria & 2 & $5 \%$ & (41) \\
\hline 2010 & Irán & E-test & 121 & No reportada & 4 & $3 \%$ & (42) \\
\hline 2010 & China & E-test & 164 & $\begin{array}{l}\text { Primaria y } \\
\text { secundaria }\end{array}$ & 2 & $1,20 \%$ & (43) \\
\hline 2010 & Irán & E-test & 132 & No reportada & 4 & $9 \%$ & (44) \\
\hline 2011 & Malasia & E-test & 187 & Primaria & 0 & 0 & (45) \\
\hline 2009 & India & ADM & 63 & No reportada & 0 & 0 & (46) \\
\hline Tasa resi & cia total de & & 2039 & & 97 & $4,75 \%$ IC :(4\%-7\%) & \\
\hline
\end{tabular}


Continuación tabla 2

\begin{tabular}{|c|c|c|c|c|c|c|c|}
\hline Año & $\begin{array}{l}\text { País de } \\
\text { origen }\end{array}$ & M étodo usado & $\begin{array}{l}\text { No. de } \\
\text { muestras }\end{array}$ & $\begin{array}{l}\text { Tipos de } \\
\text { resistencia }\end{array}$ & $\begin{array}{l}\text { Muestras } \\
\text { resistentes }\end{array}$ & $\begin{array}{l}\% \text { de resistencias } \\
\text { IC: } 95 \%\end{array}$ & R ef. \\
\hline \multicolumn{8}{|c|}{ E uropa } \\
\hline \multirow{4}{*}{2008} & \multirow{4}{*}{ Bulgaria } & \multirow{4}{*}{ ADM } & ANT 592 & \multirow{4}{*}{$\begin{array}{l}\text { Primaria y } \\
\text { secundaria }\end{array}$} & ANT 26 & ANT $4,4 \%$ & \multirow{4}{*}{ (47) } \\
\hline & & & & & & & \\
\hline & & & NNT 73 & & NNT 2 & NNT $2,7 \%$ & \\
\hline & & & AT 90 & & AT 12 & AT $13,3 \%$ & \\
\hline \multirow{3}{*}{2009} & \multirow{3}{*}{ Bulgaria } & \multirow{3}{*}{ ADM } & ANT 744 & \multirow{3}{*}{$\begin{array}{l}\text { Primaria y } \\
\text { secundaria }\end{array}$} & ANT 33 & ANT $4,4 \%$ & \multirow{3}{*}{$(47)$} \\
\hline & & & NNT 103 & & NNT 2 & NNT $1,9 \%$ & \\
\hline & & & A T 123 & & A T 13 & AT $10,6 \%$ & \\
\hline 2005 & A lemania & E-test & 150 & No reportada & 8 & $12 \%$ & (17) \\
\hline 2000 & Portugal & E-test & 394 & No reportada & 0 & 0 & (49) \\
\hline 1998 & A lemania & E-test & 271 & No reportada & 0 & 0 & $(50)$ \\
\hline 2010 & Francia & E-test & 530 & No reportada & 0 & 0 & $(51)$ \\
\hline 2011 & Finlandia & E-test & 505 & Primaria & 1 & $5 \%$ & $(52)$ \\
\hline 1999 & Italia & E-test & 206 & Primaria & 29 & $14 \%$ & (53) \\
\hline 2001 & E spaña & E-test & 140 & Primaria & 1 & $0,70 \%$ & (54) \\
\hline 1999 & Holanda & E-test & 231 & No reportada & 0 & 0 & (55) \\
\hline 2006 & M adrid & ADM & 36 & N o reportada & 0 & 0 & (56) \\
\hline 2009 & Polonia & E-test & 142 & No reportada & 0 & 0 & (57) \\
\hline 2009 & A lemania & E-test & 1118 & $\begin{array}{l}\text { Primaria y } \\
\text { secundaria }\end{array}$ & 1 & $<0,1 \%$ & (58) \\
\hline 2005 & Inglaterra & E-test & 1006 & No reportada & 18 & $1,80 \%$ & (59) \\
\hline \multicolumn{3}{|c|}{ Tasa de resistencia total de E uropa } & 6454 & & 146 & $2,26 \%$ IC $:(2 \%-3 \%)$ & \\
\hline \multicolumn{3}{|c|}{$\begin{array}{l}\text { Tasa total de resistencia de H. pylori a } \\
\text { tetraciclina mundial }\end{array}$} & 10083 & & 289 & $2,80 \%$ & \\
\hline
\end{tabular}


Tabla 3: M utaciones asociadas a la resistencia de tetraciclina

\begin{tabular}{|c|c|c|c|c|c|c|c|}
\hline Año & $\begin{array}{l}\text { País de } \\
\text { origen }\end{array}$ & $\begin{array}{l}\text { M étodo } \\
\text { usado }\end{array}$ & $\begin{array}{c}\text { No } \\
\text { M uestras }\end{array}$ & $\begin{array}{l}\text { No } \\
\text { Resistentes }\end{array}$ & $\begin{array}{l}(\%) \text { de } \\
\text { resistencia }\end{array}$ & Probable mutación & Ref. \\
\hline \multicolumn{8}{|c|}{ NORTE AMERICA } \\
\hline \multirow{6}{*}{2005} & \multirow{6}{*}{ USA } & & \multirow{6}{*}{227} & \multirow{6}{*}{8} & \multirow{6}{*}{$4 \%$} & 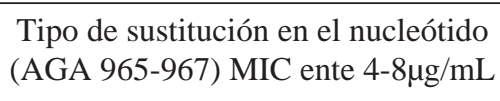 & \multirow{6}{*}{ (24) } \\
\hline & & DNA ADM & & & & A 965G 5 A islamientos & \\
\hline & & Sens. MIC & & & & A 967C 2 A islamientos & \\
\hline & & $>1 \mathrm{mg} / \mathrm{L}$ & & & & A 967T 1 A islamiento & \\
\hline & & & & & & A 965T 2 aislamiento & \\
\hline & & & & & & Ningún cambio 2 A islamientos & \\
\hline
\end{tabular}

SUR AMERICA Y CENTRO AMERICA

\begin{tabular}{|c|c|c|c|c|c|c|c|}
\hline \multirow{5}{*}{2010} & \multirow{5}{*}{ Chile } & \multirow{2}{*}{\multicolumn{2}{|c|}{ PCR-RFLP }} & \multirow{5}{*}{11} & \multirow{5}{*}{$26.8 \%$} & $\begin{array}{c}\text { Mutación en } 16 \mathrm{~S} \text { rDNA con un rango } \\
\text { de MIC de } 2 \mathrm{mg} / \mathrm{L}\end{array}$ & \multirow{5}{*}{ (27) } \\
\hline & & & & & & A 928C 1 A islamiento & \\
\hline & & Sens. MIC > & 41 & & & A G926-927 GT 6 A islamientos & \\
\hline & & & & & & A GA $926-928$ GGC & \\
\hline & & & & & & 4 A islamientos & \\
\hline \multirow{5}{*}{2008} & \multirow{4}{*}{ Cuba } & $A D M, P C R-$ & \multirow{4}{*}{28} & \multirow{4}{*}{3} & \multirow{4}{*}{$10.7 \%$} & $\begin{array}{c}\text { Mutación en } 16 \mathrm{~S} \text { rDNA con un rango } \\
\text { de MIC de } 2 \mathrm{mg} / \mathrm{L}\end{array}$ & \multirow{4}{*}{$(60)$} \\
\hline & & \multirow{3}{*}{$\begin{array}{l}\text { Sens. M IC } \\
>1 \mathrm{mg} / \mathrm{L}\end{array}$} & & & & $A 926 G$ & \\
\hline & & & & & & A $926 \mathrm{~T}$ & \\
\hline & & & & & & A $928 \mathrm{~T}$ & \\
\hline & & & & \multicolumn{2}{|c|}{ EUROPA } & & \\
\hline \multirow{3}{*}{2009} & \multirow{3}{*}{ A lemania } & RT-PCR & \multirow{3}{*}{1118} & \multirow{3}{*}{1} & \multirow{3}{*}{$<0.1 \%$} & & \multirow{3}{*}{ (58) } \\
\hline & & E-test & & & & M utación en el gen $16 \mathrm{~S}$ rDNA & \\
\hline & & $\begin{array}{l}\text { Sens. MIC > } \\
\quad 1 \mathrm{mg} / \mathrm{L}\end{array}$ & & & & GA 927-928TC & \\
\hline \multirow{3}{*}{2005} & \multirow{3}{*}{ Inglaterra } & RT-PCR & \multirow{3}{*}{1006} & \multirow{3}{*}{3} & \multirow{3}{*}{$0.2 \%$} & & \multirow{3}{*}{ (59) } \\
\hline & & E-test & & & & M utación en el gen $16 \mathrm{~S}$ rDNA & \\
\hline & & $\begin{array}{l}\text { Sens. M IC > } \\
\quad 4 \mathrm{mg} / \mathrm{L}\end{array}$ & & & & A 926G 2 A islamientos M IC 4 mg/L & \\
\hline
\end{tabular}




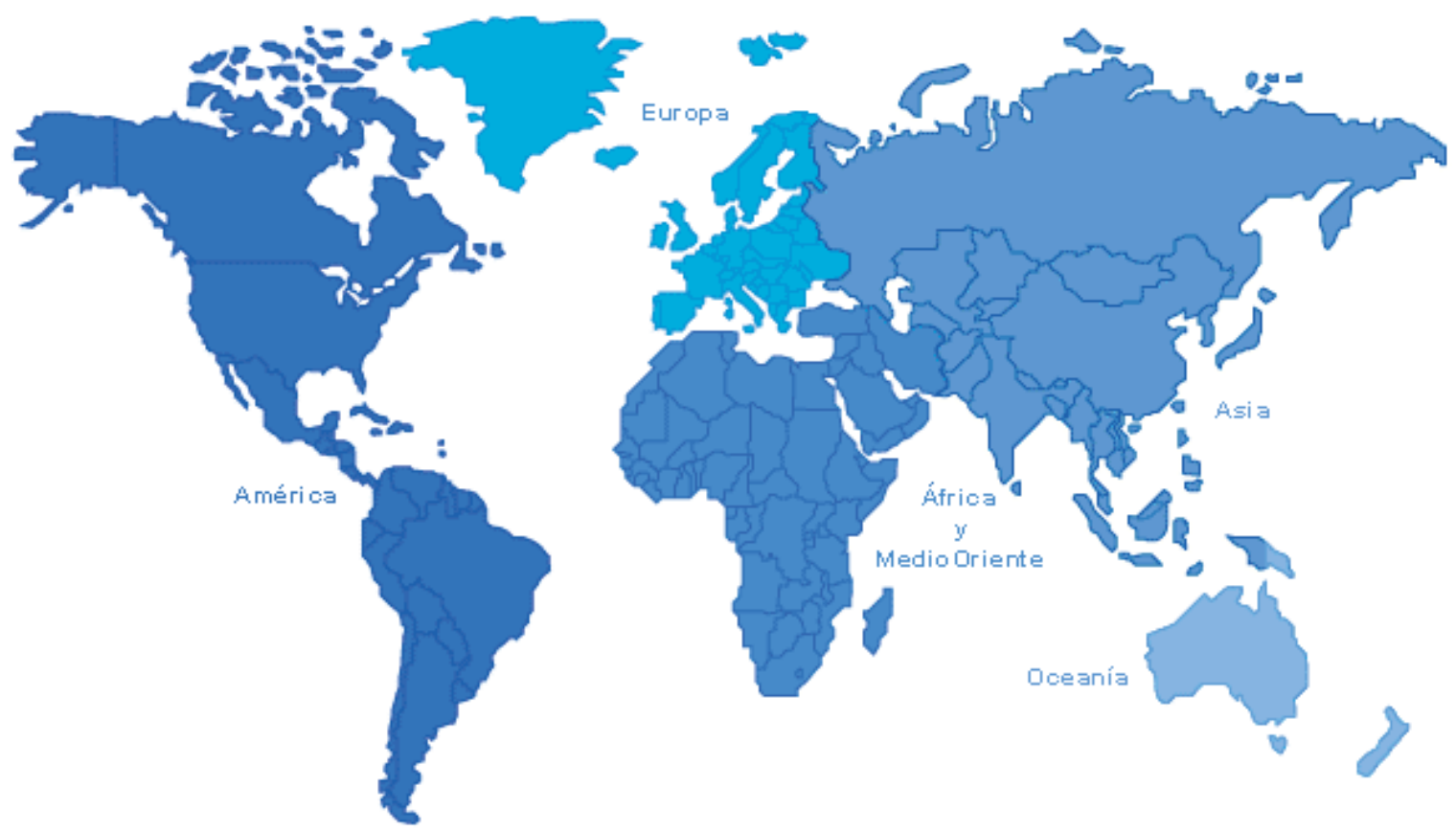

Figura 3. Prevalencia de la resistencia mundial de $\mathrm{H}$. pylori frente a tetraciclina.

T abla 4: Resistencia mundial primaria de tetraciclina.

\begin{tabular}{|c|c|c|c|c|c|c|c|}
\hline Año & $\begin{array}{l}\text { PAIS DE } \\
\text { ORIGEN }\end{array}$ & $\begin{array}{l}\text { M étodo } \\
\text { usado }\end{array}$ & $\begin{array}{c}\text { No } \\
\text { M uestras }\end{array}$ & $\begin{array}{l}\text { Tipo de } \\
\text { R esistencia }\end{array}$ & $\begin{array}{l}\text { Cepas } \\
\text { resistentes }\end{array}$ & $\begin{array}{l}(\%) \text { de } \\
\text { resistencia }\end{array}$ & Ref. \\
\hline 2006 & USA & ADM & 352 & Primaria & 0 & 0 & (25) \\
\hline 2010 & Chile & $A D M, P C R$ & 41 & Primaria & 11 & $26,8 \%$ & (27) \\
\hline 2007 & Chile & ADM & 41 & Primaria & 11 & $26,8 \%$ & (28) \\
\hline 2010 & China & ADM & 293 & Primaria & 1 & $2,93 \%$ & (37) \\
\hline 2010 & Corea & $A D M-P C R$ & 222 & Primaria & 57 & $25,7 \%$ & (38) \\
\hline 2011 & China & E-test & 73 & Primaria & 0 & 0 & (40) \\
\hline 2011 & Malasia & E-test & 187 & Primaria & 0 & 0 & (45) \\
\hline \multirow{2}{*}{2008} & \multirow{2}{*}{ Bulgaria } & \multirow{2}{*}{ ADM } & ANT 592 & \multirow{2}{*}{$\begin{array}{l}\text { Primaria y } \\
\text { secundaria }\end{array}$} & ANT 26 & ANT $4.4 \%$ & \multirow{2}{*}{ (47) } \\
\hline & & & NNT 73 & & NNT 2 & NNT $\quad 2.7 \%$ & \\
\hline \multirow{2}{*}{2009} & \multirow{2}{*}{ Bulgaria } & \multirow{2}{*}{ ADM } & ANT 744 & \multirow{2}{*}{$\begin{array}{l}\text { Primaria y } \\
\text { secundaria }\end{array}$} & ANT 33 & ANT $4.4 \%$ & \multirow{2}{*}{ (48) } \\
\hline & & & NNT 103 & & NNT 2 & NNT $1.9 \%$ & \\
\hline 2011 & Finlandia & E-test & 505 & Primaria & 1 & $5 \%$ & (52) \\
\hline 1999 & Italia & E-test & 206 & Primaria & 29 & $14 \%$ & (53) \\
\hline 2001 & España & E-test & 140 & Primaria & 1 & $0.7 \%$ & (54) \\
\hline \multirow{2}{*}{\multicolumn{3}{|c|}{ Tasa de resistencia M undial primaria }} & \multirow{2}{*}{3572} & & \multirow{2}{*}{185} & $5,1 \%$ & \\
\hline & & & & & & IC $95 \%(4 \%-6 \%)$ & \\
\hline
\end{tabular}


TABLA 5: Resistencia Secundaria M undial de tetraciclina.

\begin{tabular}{|c|c|c|c|c|c|c|c|}
\hline Autor & Año & $\begin{array}{l}\text { País de } \\
\text { origen }\end{array}$ & $\begin{array}{l}\text { M étodo } \\
\text { usado }\end{array}$ & $\begin{array}{c}\text { No } \\
\text { Muestras }\end{array}$ & $\begin{array}{c}\text { Tipo de } \\
\text { Resistencia }\end{array}$ & $\begin{array}{c}\text { Tet R } \\
\text { No }\end{array}$ & $\begin{array}{c}\text { Tet R } \\
(\%)\end{array}$ \\
\hline Sharara, A et al (34) & 2001 & Líbano & E-test & 54 & Secundaria & 1 & $2 \%$ \\
\hline M atsushima, $\mathrm{M}$ et al (35) & 2006 & Japón & ADM & 25 & Secundaria & 0 & 0 \\
\hline Shokrzadeh, L et al (41) & 2011 & Irán & $A D M-P C R$ & 42 & Secundaria & 2 & $5 \%$ \\
\hline Boyanova, L et al (47) & 2008 & Bulgaria & ADM & А Т 90 & $\begin{array}{l}\text { Primaria y } \\
\text { secundaria }\end{array}$ & 12 & A T $13,3 \%$ \\
\hline Boyanova, L et al (48) & 2009 & Bulgaria & ADM & AT 123 & $\begin{array}{l}\text { Primaria y } \\
\text { secundaria }\end{array}$ & 13 & A T $10,6 \%$ \\
\hline \multicolumn{4}{|c|}{ Tasa de resistencia M undial secundaria } & 334 & & 28 & $\begin{array}{c}8,4 \% \\
\text { IC } 95 \% \\
(5 \%-11 \%) .\end{array}$ \\
\hline
\end{tabular}

se evidenció que una sustitución simple del nucleótido A 965C o A 965T y A 967C o A 967T, estaban asociadas a la reducción de tamaño del sitio de unión de tetraciclina en la subunidad 30 S ribosomal (24).

En los estudios consultados, se describen que al tos niveles de resistencia a tetraciclina están relacionados con una sustitución completa de la tripleta A GA (926-928)-->TTC, induciendo val ores de CM I de hasta $64 \mathrm{mg} / \mathrm{L}$ y sustituciones dobles con valores de CM I de hasta 8mg/L. Las sustituciones puntuales presentan valores menores 0 iguales a $4 \mathrm{mg} / \mathrm{L}$ de CM I relacionadas con bajas resistencias en la misma región como se mencionó anteriormente $(60,27)$. Sin embargo, en los experimentos de mutaciones, ninguna cepa mostró la mutación de la tripleta compl eta. Es importante destacar que en la mayoría de estudios se evidenciaron mutaciones en sólo uno o dos nucl eótidos, dando como resistencia val ores menores 0 iguales a 4mg/L, evidenciando así, sensibilidad al antibiótico, también es importante indicar que en la tabla 3 , donde se indican las mutaciones asociadas a la resistencia de tetraciclina, la variacion de las resistencias de $\mathrm{H}$. pylori frente a tetraciclina varia de forma radical en los diferentes continentes, indicando la importancia de estudios locales que indiquen sensibilidades de los micoorganismos y mutaciones relacionadas.

En los estudios realizados para determinar las mutaciones que se relacionan con la resistencia de tetraciclina, se tomaron como referencia valores de CMI >1mg/L, para determinar los valores de CM I en bajas concentraciones. Sin embargo, en la literatura consultada los rangos son: para resistencia $\geq$
$4 \mathrm{mg} / \mathrm{L}$ y para sensibilidad 0,25- $2 \mathrm{mg} / \mathrm{L}$ (2). Esto se realizó con el fin de comprobar que las mutaciones en uno 0 dos nucl eótidos, efectivamente generaban resistencias en bajas concentraciones con valores $>1 \mathrm{mg} / \mathrm{L}$ (Tabla 3 ).

En las tablas 4 y 5 se clasificaron los artícul os que informaban que tipo de resistencia a tetraciclina habían evaluado (primaria o secundaria) con valores globales de 5,1\% (IC 95\% 4-6\%) y $8,4 \%$ (IC $95 \% 5-11 \%$ ) respectivamente. Se demuestra así que una de las razones de aparición de resistencia a tetraciclina está asociada con tratamiento previo con este antibiótico, generando que $\mathrm{H}$. pylori presente mutaciones de resistencia a tetraciclina. Sin embargo, la mayoría de artícul os consultados no describían si esta resistencia era primaria o secundaria ya que los pacientes entrevistados no recuerdan que antibióticos han tomado previamente 0 tampoco se puede determinar si este fue prescrito para otro tipo de infección. la falta de este tipo de información impide que la investigación acerca de mutaciones post tratamiento se pueda explicar ampliamente.

Como se demuestra en esta revisión, la resistencia de H . pylori a tetraciclina es muy baja en la mayoría de los países, siendo el valor más al to $8,4 \%$, encontrado en Centro y Sur A mérica. No obstante la baja tasa de resistencia, la utilización de este antimicrobiano se limita a los tratamientos de rescate como terapia cuádruple con bismuto, de acuerdo a la recomendación del último consenso de M aastricht en 2007 (11). En nuestro medio hace una década se demostró eficacia de $80 \%$ de un esquema de rescate como terapia concomitante, la cual incluía tetraciclina (terapia cuádruple sin bismuto) (61). 
Dada la complejidad de los esquemas de terapias cuádruples con bismuto, se ha ensayado la utilización de omeprazol dos veces al día, mas una cápsula tres veces al día que contiene tetraciclina, metronidazol y bismuto (Pylera $\left.{ }^{\circledR}\right)$, logrando tasas de éxito por encima del $90 \%$ (62). Teniendo en cuenta que a nivel mundial, son muy bajas las tasas de éxito con las terapias triples que utilizan metronidazol, claritromicina o levofloxacina, no superando el 80\% (63) y que en el medio colombiano son inferiores a este valor, sería de suma importancia, para Colombia investigar la tasa de resistencia a la tetraciclina y si se demuestra que es muy baja, al igual que la amoxicilina (2,6\%), pudiera incluirse en terapias al ternas, bien sea en la forma de administración como el Pylera ${ }^{\circledR} 0$ diseñando nuevos esquemas con este medicamento administrado dos veces al día, teniendo en cuenta que su vida media plasmática es de seis horas (64).

\section{Conclusiones}

Los estudios revisados demuestran tasas de prevalencia de resistencia de Helicobacter pylori de $1 \%$ para Norte A mérica, $8 \%$ para Centro y Sur A mérica; $5 \%$ para A sia y $2 \%$ para Europa. Las mutaciones más reportadas fueron A 926G y A 926T y sólo se encontró una sustitución del codón A GA 926-928G GC y en ningún aislamiento de los incluidos en la revisión se encontró la mutación responsable de al ta resistencia A GA 926-928TTC. Se evidenció que la tasa de resistencia a tetraciclina es mayor cuando la administración del antibiótico es en un segundo tratamiento, indicando la importancia del control de la venta de antibióticos.

\section{Agradecimientos}

Al grupo de Helicobacter pylori, a Jenny Á vila y Liliana Rosero, por su colaboración, consejos, apoyo y asesoría.

\section{Financiación}

Este trabajo fue financiado por COL CIEN CIAS proyecto código 120340820464.

\section{Conflicto de intereses}

Los autores declaran no tener conflicto de intereses con respecto a este estudio.

\section{R eferencias}

1. Ricci V, Romano M, B oquet P. M olecular cross-talk between $\mathrm{H}$ elicobacter pylori and human gastric mucosa. World J ournal of G astroenrology 2011; 17 (11): 1383-1399.
2. B rown LM . Helicobacter pylori: epidemiology and routes of transmission. Epidemiologic Reviews 2000; 22 (2): 283-297.

3. Blaser M J, A therton JC. H elicobacter pylori persistence: biology and disease. The J ournal of Clinical Investigation. 2004; 113 (3): 321-333.

4. Wroblewski LE, Peek RM Jr, Wilson KT. H elicobacter pylori and gastric cancer: factors that modulate disease risk. Clinical M icrobiology Reviews 2010; 23 (4): 713-739

5. Y amaoka Y. M echanisms of disease: Helicobacter pylori virulence factors. Nature Reviews Gastroenterology \& Hepatology 2010; 7 (11): 629-641.

6. M inisterio de la Protección Social, Instituto $\mathrm{N}$ acional de Cáncer. A nuario estadístico 2009. República de Colombia 2011.

7. A saka M, K ato M, Graham DY. Prevention of Gastric Cancer by $\mathrm{Helicobacter}$ pylori Eradication. Internal M adicine 2010; 49 (7):633-636.

8. Mera $R$, Fontham ET, Bravo LE, Bravo JC, Piazuelo $M B, C$ amargo M C, and Correa P. Long term follow up of patients treated for $\mathrm{H}$ elicobacter pylori infection. Gut. 2005; 54:1536-1540.

9. Chuah SK, Tsay FW, Hsu PI, Wu DC. A new look at anti-Helicobacter pylori therapy. World J ournal of Gastroenterology 2011; 17 (35): 3971-3975.

10. Selgrad $M, M$ alfertheiner $P$. Treatment of $\mathrm{H}$ elicobacter pylori. Current 0 pinion in Gastroenterology 2011; 27: 565-570

11. $M$ alfertheiner $P, M$ egraud $F, O^{\prime} M$ orain $C, B$ azzoli $F, E I-$ Omar E, Graham D, Hunt R, Rokkas T, V akil N, K uipers EJ, The European Helicobacter Study Group (EHSG). Current concepts in the management of Helicobacter pylori infection: the Maastricht III Consensus Report. Gut 2007; 56, 772-781.

12. Latorre R, Gallo G. Helicobacter pylori: su importancia practica en la gastroenterología. Revista M edica Clinica Los Condes 2008; 19 (4) 323-329.

13. Kim JJ, Reddy R, Lee M, Kim J G, El-Zaatari FA, Osato M S, Graham DY , K won DH. A nalysis of metronidazole, clarithromycin and tetracycline resistance of H elicobacter pylori isolates from Korea. Journal of Antimicrobial Chemotherapy. 2001; 47: 459-461.

14. De Francesco V, Zullo A, Hassan C, Giorgio F, Rosania $R$, Ierardi $E$. Mechanisms of Helicobacter pylori antibiotic resistance: An updated appraisal. World J ournal of Gastrointestinal Pathophysiology 2011; 15; 2 (3): 35-41. 
15. M egraud $F$, Lehn $N$, Lind $T$, Bayerdorffer $E, O$ 'morain $C$, Spiller R, Unge $P$, van Zanten SV, W rangstadh $M$, and Burman CF. A ntimicrobial Susceptibility testing of $\mathrm{H}$ elicobacter pylori in a large multicenter trial: the $\mathrm{M} \mathrm{ACH}$ 2 study. J ournal of Antimicrobial Chemotherapy 1999; 43, 2747-2752.

16. A noushiravani M, Falsafi T, Niknam V. Proton motive force-dependent efflux of tetracycline in clinical isolates of Helicobacter pylori. J ournal of M edical M icrobiology 2009; 58, 1309-1313.

17. Glocker E, B erning M, Gerrits M M, K usters JC and K ist M. Real-time PCR screening for $16 S$ rRNA mutations associated with resistance to tetracycline in $\mathrm{Hel}$ icobacter pylori. J ournal of Antimicrobial Chemotherapy 2005; 49, 3166-3170.

18. N onaka L, Connell SR, Taylor D. 16 S rR N A mutations that confer tetracycline resistance in $\mathrm{H}$ elicobacter pylori decrease drug binding in Escherichia coli ribosomes. J ournal of Bacteriology 2005; 187, 3708-3712.

19. Trieber $C A$, Taylo DE. M utations in the 165 rRNA Genes of $\mathrm{H}$ elicobacter pylori mediate resistance to tetracycline. J ournal of Bacteriology 2002; 184 (8): 2131-140.

20. De Francesco V , Giorgio F, H assan C, M anes $G, V$ annella L, Panella C, Ierardi E, Zullo A. W orldwide H. pylori A ntibiotic resistance: a systematic review. J ournal of Gastrointestinal and Liver D iseases 2010; 19 (4): 409-414.

21. Á vila JM, Rey M, M ercado M M, Villamizar OR, Otero W, Trespalacios A. Comparación de las pruebas de dilución en Agar y PCR para determinación de susceptibilidad antimicrobiana de Helicobacter pylori. Revisión sistemática de la literatura. Revista Colombiana de $\mathrm{G}$ astroenter ología 2009; 116-127.

22. Rey $M$, A vilaJ M, Trespalacios A A, Villamizar OR, Otero W, M ercado M M. Sensibilidad y especificidad de E-test para la determinación de susceptibilidad antimicrobiana en aislamientos clínicos de $\mathrm{H}$ elicobacter pylori. NOVA. 2008; 6(6): 220-229.

23. Jaeschke. R, G ordon. H, Guyatt, M D y Sackett. DL. Guías para usuarios de la literatura médica, parte $B$ ¿Cuáles son los resultados?, ¿me ayudarán a la asistencia de mis pacientes? J ournal of American Medical Association 1997; 703-707.

24. Y ih Wu J, Kim JJ, Reddy R, Wang W M, Graham DY, $\mathrm{K}$ won DH. Tetracycline-resistant Clinical Helicobacter pylori I solates with and without mutations in $16 \mathrm{~S}$ rR NAencoding genes. Antimicrobial Agents and Chemotherapy 2005; 49 (2): 578-583.

25. B ruce M G, Bruden DL, M CM ahon BJ, Hennessy TW, Reasonover A, M orris J , Hurlburt D A, Peters H, Sacco F,
M artinez $P$, Swenson $M, B$ erg DE, Parks $D$ y Parkinson A J. A laska sentinel surveillance for antimicrobial resistance in $\mathrm{H}$ elicobacter pylori Isolates from A laska native persons, 1999-2003. Helicobacter 2006 11: 581-588.

26. Tveit $A H, B$ ruce $M G, B$ ruden $D L, M$ orris J, Reasonover A, Hurl burt DA, Hennessy TW, y M CM ahon B. A laska sentinel surveillance study of $\mathrm{H}$ elicobacter pylori isolates from A laska native persons from 2000 to 2008. J ournal of Clinical M icrobiology 2011; 49 (10) 3638-3643.

27. Toledo H, y López -Solís R. Tetracycline resistance in Chilean clinical isolates of Helicobacter pylori. Journal of Antimicrobial and Chemotherapy 2010; 65: 470-473.

28. V allejos C, Garrido L, Cáceres D, M adrid A M , D efilippi C, D efilippi C, Toledo H. Prevalencia de la resistencia a metronidazol, claritromicina y tetraciclina en $\mathrm{H}$ elicobacter pylori aislado de pacientes de la región metropolitana. Revista M édica de Chile 2007; 135: 287-93.

29. Llanes R, Soria C, Nagashima S, K obayashi N, Gala A, Guzmán $D$, F eliciano $O, V$ al dés $L$, Gutiérrez $O$, F ernández $H$, Llop A, y Wada A. Phenotypic and genetic characterization of antimicrobial profiles of $\mathrm{H}$ elicobacter pylori strains in Cuba. J ournal of H ealth, P opulation and Nutrition 2010; 28(2):124-129.

30. Á lvarez A; M oncayo JI, Santacruz J J, Corredor LF, Reinosa $E, M$ artínez JW, B eltrán L. Resistencia a metronidazol y claritromicina en aislamientos de Helicobacter pylori de pacientes dispépticos en Colombia. Revista Médica de Chile 2009; 137: 1309-1314.

31. García GT, A randa KRS, Goncalves MEP, Cardoso SR, Iriya $K$, Silva NP, Scaletsky ISA. High prevalence of clarithromycin resistance and cagA, vacA, iceA 2 , and babA 2 genotypes of $\mathrm{Helicobacter}$ pylori in Brazilian children. J ournal of Clinical M icrobiology 2010; 48(11): 4266-4268.

32. Ortiz AP, Lima M, Borges YH, Vitiello L, Bueno MC, $M$ endonça $S$, y Pedrazzoli Jr J. A nalysis of antimicrobial susceptibility and virulence factors in H elicobacter pylori clinical isolates. B M C Gastroenter ology 2003; 3 (20): 1-6.

33. Debets-O ssenkopp $Y$, Reyes G, Mulder J, Stegge BM, Peters J, Savelkoul 1 PHM, Tanca J, Peña S, $\checkmark$ andenbroucke-Grauls C. Characteristics of clinical Helicobacter pylori strains from Ecuador. J ournal of Antimicrobial Chemotherapy 2003; 51: 141-145

34. Sharara A, Chedid M, A raj GF, Barada KA, Mourad $\mathrm{FH}$. Prevalence of Helicobacter pylori resistance to metronidazole, clarithromycin, amoxycillin and tetracycline 
in L ebanon. International J ournal of Antimicrobial Agents 2002; 19: 155- 158

35. M atsushima $M$, Suzuki $T$, K urumada $T$, Watanabe $S$, WatanabeK, K obayashi K, Deguchi R, M asui A, Takagi A, Shirai T, M uraoka H, K obayashi I, y M ineT. Tetracycline, metronidazole and amoxicillin-metronidazole combinations in proton pump inhibitor-based triple therapies are equally effective as alternative therapies against $\mathrm{H}$ elicobacter pylori Infection. J ournal of Gastroenterology and H epatology 2006; 21: 232-236.

36. K hedmat $H, A$ mini $M$, Jafari $A M, A$ fshar $F N$, Soltanpoor MJ, Fallahian F, Izadi M, y Hosseini M S. In vitro susceptibility testing of $\mathrm{Helicobacter}$ pylori to metronidazole, amoxicillin, tetracycline, and ciprofloxacin in Iran. Research J ournal of Microbiology 2007; 2:12 947-953.

37. Sun QJ, Liang X, Zheng Q, Gu W Q, Liu W Z, X iao SD, Lu $H$. Resistence of $H$ elicobacter pilory to antibiotics from 2000 to 2009 in Shangai. Word J ournal of G astroenterology 2010; 16 (40): 5118-2840.

38. Hwang TJ, Kim N, Kim HB, Hwan Lee B, Nam RH, Park JH, K young L ee M, Park Y S, Lee D H, Jung HC,y Song IS. Change in A ntibiotic R esistance of H elicobacter pylori Strains and the Effect of A 2143G Point M utation of $23 \mathrm{~S}$ rR NA on the Eradication of $\mathrm{H}$. pylori in a Single Center of K orea. J ournal of Clinical Gastroenterology 2010; 44(8):536-43.

39. Samra Z, Shmuely H, Niv Y, Dinari G, Passaro DJ, Geler A, Gal E, Fishman M , B achor J, y Y ahav J. Resistance of H elicobacter pylori Isolated in Israel To metronidazole, Clarithromycin, tetracycline, amoxicillin, and cefixime. J ournal of Antimicrobial Chemotherapy 2002; 49: 10231026.

40. Liu G, Xu X, He L, Ding Z, Gu Y, Zhang J, y Zhou L. Primary A ntibiotic Resistance of Helicobacter pylori Isolated from B eijing Children. H elicobacter 2011; 16: 356-362.

41. Shokrzadeh L, Jafari F, Dabiri H, Baghaei K, Zojaji H, A lizadeh AH, M ehdi A slani M, Zali M R. Antibiotic susceptibility profile of Helicobacter pylori isolated from the dyspepsia patients in Theran, I ran. The Saudi J ournal of G astroenterology 2011; 17: 4. 261-264.

42. Farshad S A Iborzi A Japoni A Ranjbar R, A sl KH, B adiee P, Shahidi M A, Hosseini M . A ntimicrobial susceptibility of $\mathrm{Helicobacter}$ pylori strains isolated from patients in Shiraz, Southern I ran. J ournal of Clinical G astroenterology 2010; 16(45): 5746-5751.

43. Gao W, Cheng H, Hu F, Li J, Wang L, Y ang G, XuL y Zheng $X$. The Ev volution of Helicobacter pylori A ntibiotics
Resistance over $10 Y$ ears in B eijing, China. Helicobacter 2010; 15: 460-466

44. Talebi B ezmin A, M obarez A M, Taghvaei T y W olfram L. A ntibiotic Resistance of Helicobacter pylori in M azandaran, N orth of I ran. Helicobacter 2010; 15: 505-509

45. A hmad N, Zakaria WR and Mohamed R. A nalysis of antibiotic susceptibility patterns of Helicobacter pylori isolates from M alaysia. Helicobacter 2011; 16: 47-51

46. Singh V, M ishra S, M aurya P, Rao G, J ain A K, Dixit VK, Gulati AK y Nath $G$. D rug resistance pattern and clonality in $\mathrm{H}$. pylori strains. J ournal of Infection in D eveloping Countries 2009; 3(2):130-136

47. Boyanovaa L, Gergovaa G, Nikolovb R, Davidkovc L, $\mathrm{K}$ amburovd V, J eleveC, M itova. Prevalence and evolution of Helicobacter pylori resistance to 6 antibacterial agents over 12 years and correlation between susceptibility testing methods. D iagnostic M icrobiology and Infectious Disease 2008; 60: 409-415.

48. B oyanova L. Prevalence of multidrug-resistant H elicobacter pylori in Bulgaria. J ournal of M edical Microbiology 2009; 58: 930-935.

49. Cabrita J, Oleastro $M, M$ atos $R, M$ anhente $A, C$ abral J, B arros R, Lopes A .I, Ramalho P, N eves B.C, y Guerreiro A.S. Features and trends in $\mathrm{H}$ elicobacter pylori antibiotic resistance in Lisbon area, Portugal (1990-1999). J ournal Antimicrobial Chemotherapy 2000; 46: 1029-1031.

50. WolleK, Nilius M, L eodolter A, M üller W .A, M alfertheiner $\mathrm{P}, \mathrm{K}$ önig W. Prevalence of $\mathrm{H}$ elicobacter pylori resistance to several antimicrobial agents in a region of Germany. European J ournal of Clinical Microbiology and Infectious Diseases 1998; 17:519-521.

51. Raymond J, Lamarque D, K alach N, Chaussade S, $\mathrm{B}$ urucoa C. High level of antimicrobial resistance in french Helicobacter pylori isolates. Helicobacter 2010; 15: 21-27.

52. K ostamo P, V eijola L, O ksanen A, Sarna S, Rautel in $H$. Recent trends in primary antimicrobial resistance of Helicobacter pylori in Finland. International J ournal of Antimicrobial Agents 2011; 37: 22-25

53. Realdi $G, M$ aria $P, D$ ore M P, Piana A, A tzei A, Carta $M$, Cugia $L, M$ anca $A$, A re B M, M assarelli $G$, M ura I, M aida A, y Graham DY. Pretreatment antibiotic resistance in Helicobacter pylori infection: results of three randomized controlled studies. Hel icobacter 1999; 2(4):106-112.

54. Cuchí E, FornéM, QuintanaS, LiteJ y Garau J. Evolución de la sensibilidad de 235 cepas de Helicobacter pylori entre 1995 y 1998 e impacto del tratamiento antibiótico. 
Enfermedades I nfecciosas y M icrobiología C línica 2002; 20(4):157-160.

55. Debets-O ssenkopp Y , Herscheid A J, R aymond G. J , Kuipers EJ, K usters J G y V andenbroucke-G rauls C. Prevalence of Helicobacter pylori resistance to metronidazole, clarithromycin, amoxycillin, tetracycline and trovafloxacin in The Netherlands. J ournal of A ntimicrobial C hemotherapy 1999; 43(4): 511-515.

56. Díaz-Regañón J, A larcón T, Domingo D y López-B rea M. Sensibilidad de 36 aislamientos de $\mathrm{H}$ elicobacter pylori a cuatro antibióticos de primera línea y características de virulencia. Revista Española de Q uimioterapia 2006; 19 (1): $34-38$

57. A ndrzejewska E, Szkaradkiewicz A, Karpinski T. A ntimicrobial resistance of $\mathrm{Helicobacter}$ pylori clinical Strains in the last $10 Y$ ears. Polish J ournal of M icrobiology. 2009; 58 (4): 301-305.

58. Wueppenhorst N, Stueger HP, K ist M and Glocker E. Identification and molecular characterization of triple- and quadruple-resistant Helicobacter pylori clinical isolates in Germany. Journal of Antimicrobial Chemotherapy 2009; 63: 648-653.

59. Lawson AJ, Elviss NC y O wen RJ. Real-timePCR detection and frequency of $16 \mathrm{~S}$ rDNA mutations associated with resistance and reduced susceptibility to tetracycline in $\mathrm{H}$ elicobacter pylori from England and W ales. J ournal of Antimicrobial Chemotherapy 2005; 56: 282-286

60. Torres LE, Rodríguez EE, Hernández M, M oreno A, Roblejo Y, Samada M, Cansino J, M elián K, A lonso J, M artínez M , Fando R, B ermúdez L, Rodríguez B . Eventos moleculares que tributan a la resistencia a tetraciclina en Helicobacter pylori, su detección en aislados clínicos cubanos. Revista CENIC Ciencias Biológicas 2008; 39 (2): 121-126.

61. Gutierrez O, Otero W, Cardona H. Terapia cuádruple con furazolidona como tratamiento de rescate para la infección por Helicobacter pylori. Revista Colombiana de Gastroenterologia 2003; 18: 222-228

62. Saleem A, Qasim A, O'Connor HJ, O'M orain CA. Pylera for the eradication of Helicobacter pylori infection. Expert Review of Anti-infective Therapy 2009; 7:793-799.

63. Otero W. Trespalacios A A, Otero E. Helicobacter pylori: tratamiento actual. Un importante reto en gastroenterología. Revista Colombiana de G astroenterologia 2009; 24:279292

64. Furuta T, Graham DY. Pharmacologic aspects of eradication therapy for Helicobacter pylori infection. Gastroenterology Clinics of North A merica 2010; 39: 465-480. 